\title{
Improving Retention in Care and Promoting Adherence to HIV Treatment: Protocol for a Multisite Randomized Controlled Trial of Mobile Phone Text Messaging
}

Elvis Asangbeng Tanue ${ }^{1}, \mathrm{MSc}$; Dickson Shey Nsagha ${ }^{1}, \mathrm{PhD}$; Nana Njamen Theophile ${ }^{2}$, MD; Jules Clement Nguedia $\mathrm{Assob}^{3}, \mathrm{PhD}$

\footnotetext{
${ }^{1}$ Department of Public Health and Hygiene, Faculty of Health Sciences, University of Buea, Buea, Cameroon

${ }^{2}$ Department of Obstetrics and Gynecology, Faculty of Health Sciences, University of Buea, Buea, Cameroon

${ }^{3}$ Department of Medical Laboratory Sciences, Faculty of Health Sciences, University of Buea, Buea, Cameroon
}

\section{Corresponding Author:}

Dickson Shey Nsagha, PhD

Department of Public Health and Hygiene

Faculty of Health Sciences

University of Buea

PO Box 12

Buea, 00237

Cameroon

Phone: 237677499429

Email: dsnsagha@gmail.com

\section{Abstract}

Background: The World Health Organization has prioritized the use of new technologies to assist in health care delivery in resource-limited settings. Findings suggest that the use of SMS on mobile phones is an advantageous application in health care delivery, especially in communities with an increasing use of this device.

Objective: The main aim of this trial is to assess whether sending weekly motivational text messages (SMS) through mobile phones versus no text messaging will improve retention in care and promote adherence to treatment and health outcomes among patients receiving HIV treatment in Fako Division of Cameroon.

Methods: This is a multisite randomized controlled single-blinded trial. Computer-generated random block sizes shall be used to produce a randomization list. Participants shall be randomly allocated into the intervention and control groups determined by serially numbered sealed opaque envelopes. The 156 participants will either receive the mobile phone text message or usual standard of care. We hypothesize that sending weekly motivational SMS reminders will produce a change in behavior to enhance retention; treatment adherence; and, hence, health outcomes. Participants shall be evaluated and data collected at baseline and then at 2, 4, and 6 months after the launch of the intervention. Text messages shall be sent out, and the delivery will be recorded. Primary outcome measures are retention in care and adherence to treatment. Secondary outcomes are clinical (weight, body mass index), biological (virologic suppression, tuberculosis coinfection), quality of life, treatment discontinuation, and mortality. The analysis shall be by intention-to-treat. Analysis of covariates shall be performed to determine factors influencing outcomes.

Results: Recruitment and random allocation are complete; 160 participants were allocated into 3 groups (52 in the single SMS, 55 in the double SMS, and 53 in the control). Data collection and analysis are ongoing, and statistical results will be available by the end of August 2019.

Conclusions: The interventions will contribute to an improved understanding of which intervention types can be feasible in improving retention in care and promoting adherence to antiretroviral therapy.

Trial Registration: Pan African Clinical Trial Registry in South Africa PACTR201802003035922; https://pactr.samrc.ac.za/TrialDisplay.aspx?TrialID=3035

International Registered Report Identifier (IRRID)： DERR1-10.2196/15680

(JMIR Res Protoc 2020;9(8):e15680) doi: 10.2196/15680 


\section{KEYWORDS}

adherence; antiretroviral; HIV; randomized controlled trial; retention in care; text messaging

\section{Introduction}

In patients infected with HIV, viral replication can be effectively suppressed with antiretroviral therapy (ART), allowing the body's immune system to restore and function adequately [1]. Suppressed viral replication in HIV infection has been proven to dramatically reduce mortality and morbidity rates, leading to improved quality of life and improved perceptions on HIV/AIDS from a death sentence to a manageable chronic disease [2]. Yet, the population effect of ART depends on high coverage and sustained adherence to treatment among people living with HIV [3].

The SMS of the mobile phone is an inexpensive and suitable means of communication that can be used in conveying health messages to persons who own or have access to mobile phones. In 2013, 97\% of the world's population were mobile phone subscribers [4]. Moreover, by 2015, 71 per 100 inhabitants of Cameroon were accessing mobile phones [5].

Study protocols on the use of SMS technology to promote adherence to ART have been developed in Kenya [6], India [7], and Cameroon [8]. In 2005, the World Health Organization spelled out the use of new technologies to assist health care delivery in resource-limited settings [9]. Since then, the SMS has shown promising results in improving HIV health care delivery and communication between health personnel and clients, and as an appointment reminder in trials conducted in Kenya [10], Cameroon [11,12], South Africa [13], and elsewhere [14].

The use of text messages in improving adherence to primary care has been found to be more profitable than phone calls [15]. Patient-centered mobile health (mHealth) interventions have had promising outcomes in sickle cell disease management [16]. Systematic reviews have pointed to the success of mobile phone text messaging interventions in improving medication adherence among people living with chronic diseases [17-19]. These findings suggest the use of SMS as a more advantageous application of the mobile phone in health care delivery especially in communities with increasing use of these devices.

Only a single mHealth intervention has been conducted in Cameroon and included only one hospital and 198 participants for a period of 6 months [11]. This lone Cameroonian mHealth intervention together with those conducted elsewhere $[10,13,15]$ has shown promising results on ART adherence and retention in HIV care. Thus, further research involving patients from different treatment centers, and most especially in the phase of government efforts to fight HIV/AIDS, is warranted in Cameroon. This study is timely as it is based on this strategy to improve and promote ART adherence and retention in care in Cameroon. Furthermore, the study will enhance our understanding of the extent that mHealth intervention promotes healthy behaviors and supports psychosocial well-being among patients receiving treatment for chronic diseases. Therefore, this study will contribute to an improved understanding of which text message frequency can be feasible when, why, and for whom.

The goal of this trial is to determine whether sending weekly motivational text messages through mobile phones versus no text messaging will improve retention in care and promote adherence to treatments and health outcomes among patients receiving HIV treatments over a 6-month period. We hypothesize that sending weekly motivational text message reminders will produce a change in behavior to enhance retention; treatment adherence; and, hence, health outcomes.

Participants will be recruited from two hospitals approved to provide free HIV treatment services in Fako Division of the South West Region of Cameroon.

\section{Methods}

The trial was registered with the Pan African Clinical Trial Registry [20] in South Africa on February 1, 2018. The unique identification number for the protocol is PACTR201802003035922.

\section{Study Design}

The design rests on a three-arm, randomized controlled trial with two intervention arms and one control group. The trial shall be single-blinded where the investigators and participants shall not be blinded to the intervention, whereas interviewers and data analysts will be blinded to group allocation. The intervention shall include the use of mobile cellphone SMS in addition to the standard of care provided to the clients. Participants shall be randomly allocated in a 1:1:1 ratio to one of three arms prior to the beginning of the intervention: (1) once weekly mobile phone SMS, (2) twice weekly mobile phone SMS, and (3) a control group that shall not receive mobile cellphone SMS. Clients will be recruited from the government-approved centers of the Regional Hospital Buea and Regional Hospital Limbe providing HIV treatment services.

\section{Randomization}

This is a parallel-group design evaluating the effects of adding once-weekly mobile phone text messages and twice-weekly mobile phone text messages to the usual standard of care (intervention) versus usual standard of care alone (control) among HIV clients receiving ART. Eligible and consenting participants shall be randomized to interventions and control arms using a 1:1:1 allocation ratio by the opaque sealed envelope method. A computer-generated randomization list will be produced using random block sizes of 2,4 , and 6 by the research biostatistician. The allocation codes shall then be put in serially numbered opaque sealed envelopes and administered by the research staff at the various hospitals. Trained interviewers, who shall be blinded to group allocation, will collect data using pretested questionnaires containing sociodemographic data, clinical information, retention, and adherence measures at baseline and at 2, 4, and 6 months. The biostatistician shall also be blinded to group allocation. Blood samples will be collected 
and analyzed for viral load during the trial once a participant has been on ART for at least 6 months. The time schedule of enrollment, interventions, and assessments are summarized in Figure 1.

Figure 1. Recruitment and random allocation into the text messaging and control groups. AFB: acid-fast bacilli; ART: antiretroviral therapy; TB: tuberculosis; WHO: World Health Organization.

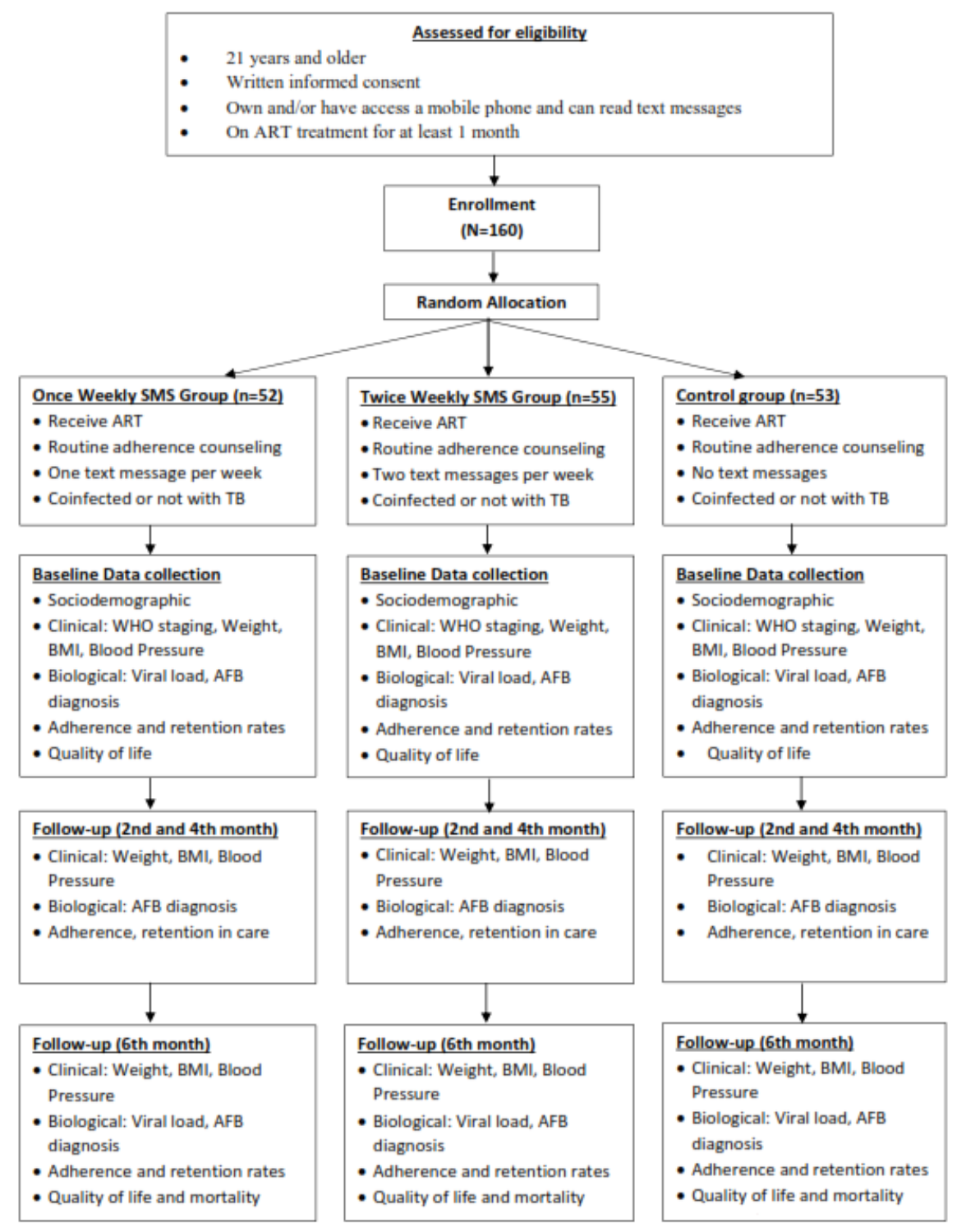

\section{Trial Setting}

Cameroon is a sub-Saharan central African country made of ten regions and a population of over 24 million inhabitants [21]. The study will be implemented in Fako Division of the South West Region of Cameroon. The adult prevalence of HIV in the country was $3.4 \%$ in 2018 [22]. The adult prevalence of HIV in the South West Region was 3.6\% in 2018 [22]. Most inhabitants practice agriculture as the main economic activity. The region has two seasons: the dry season from October to March and the wet season from April to September. Almost all ethnic groups in Cameroon are represented in the region, attracted by the fertile volcanic soil and the Cameroon Development Corporation, a giant agricultural corporation that seconds the state of Cameroon in employment. The two hospitals selected for the trial have government-approved centers offering free treatment and care services for HIV to the population of the region. Participants will be residents in their various communities and support groups while visiting these treatment hospitals for routine care during the entire study period. As per the Cameroonian Ministry of Public Health national guidelines, newly diagnosed clients are initiated on free nonnucleotide reverse transcriptase inhibitors-based ART irrespective of their viral load count or $\mathrm{CD}_{4}{ }^{+} \mathrm{T}$ cell count [11]. Recently, HIV clients initiating ART were systematically placed on isoniazid prophylaxis for the first 6 months of their ART. These are the largest treatment centers in the region and offer enormous potential for recruitment. 


\section{Participants: Inclusion and Exclusion Criteria}

The study population shall include HIV clients either coinfected with or without tuberculosis who come to the hospital for a routine health evaluation. Participants shall be consecutively recruited and randomly allocated to the intervention and control groups.

We shall include clients who are 21 years or older, have access to or own a mobile phone and can read text messages, and who have been on ART for at least a month and planned to live in Cameroon during the period of the study. We shall place more emphasis on clients who have started ART for at least 1 month to measure the differential effects for clients initiating ART versus those who have been under treatment for longer durations. Informed consent is a prerequisite for participating in the study and shall be provided orally and in writing.

We shall exclude clients who have been on ART for less than a month and who are younger than 21 years. Clients who have used ART for at least 1 month are chosen to enable us to be able to calculate a baseline figure for treatment adherence.

\section{Intervention}

The HIV clients will be receiving ART for free from the hospital staff at the HIV treatment centers. The HIV clients who shall be coinfected with tuberculosis shall also receive directly observed treatment short-course for free. This is the usual standard of care for people living with HIV/AIDS and tuberculosis in Cameroon [23]. Patients with HIV on any of these treatments shall be randomized to receive mobile phone text message reminders. The trial shall comprise 2 intervention groups and 1 control group to investigate the impact of text message reminders on retention in care and adherence to treatment and health outcomes.

A short text message will be sent to the participants in the intervention groups in English or French depending on the preferred language of each client. The content of these text messages has been developed and pretested in focus group discussions involving people living with HIV/AIDS, their caregivers, and health care workers. The construct of the health belief model was used to develop these text messages that are sociocultural acceptable and targeted at improving retention in care and promoting adherence to treatment [24]. The messages are motivating and shall act both as reminders and a cue to action. Participants unanimously agreed that the messages should not contain the name of the disease. It was also concluded that the word "food" should be used in place of medication or drug when sending the messages (Textbox 1). The message shall also contain a phone number that the participants can call back if they need directives on their treatment and care. The content shall be varied so as to retain participants' attention and interest throughout the period of the study and to explore the various aspects of behavior change. There shall be a list of phone numbers that the messages targeted at improving retention in care shall be sent midway before the next clinic visit schedule and then 2 days before each clinic visit appointment. The messages targeted at promoting adherence to treatment will be sent twice every week. The "delivery report" function of the mobile phone shall be used to verify and to record whether the messages have been delivered to the clients. Clients will be contacted through the phone numbers of their contact persons in the event of undelivered messages. The issue of missing phone numbers shall be resolved by replacing clients' existing contracts with their recent phone numbers. One message will be sent per week in the evening between 6 PM and 7 PM of a day chosen by the client in the once-weekly SMS intervention arm. In addition, two messages shall be sent per week in the evening between 6 PM and 7 PM of 2 days chosen by the client in the twice-weekly SMS intervention arm. The mobile phone text messaging shall be provided as add-ons to the usual standard of care, which includes rare ART counseling and occasional home visits.

Textbox 1. Examples of the text messages that will be sent in the intervention group.

Sample SMS to improve retention in care

- You are so loved. Handle your health with care, visit us on xx-xx-xxxx.

- You are very important. Do not forget your visit on $\mathrm{xx}-\mathrm{xx}-\mathrm{xxxx}$.

- Are you busy? This is why I remind you to come for your visit on $\mathrm{xx}-\mathrm{xx}-\mathrm{xxxx}$.

- Your health is important. Come for your appointment on Xx-XX-Xxxx.

Sample SMS to promote adherence to treatment

- Your health is important. Take your food.

- You are very important. Do not play with your health. Take your food.

- You are so loved. Handle your health with great care. Take your food.

- Are you very busy? This is why I remind you to regularly take your food.

\section{Control}

The routine practice in Cameroon is that, at ART initiation, the responsibilities of the treatment centers usually explain the side effects of medications and problems associated with poor adherence to the clients prior to dispensing drugs. All participants including those in the control arm have received this educational message during their routine hospital visits. Participants randomly allocated to the control group shall not receive mobile phone SMS. However, they shall receive ART, be screened for tuberculosis coinfection, and be interviewed alongside the other participants. 


\section{Study Objectives}

\section{Primary Objective}

The main aim of this trial is to investigate the impact of adding mobile phone SMS to the usual standard of care versus usual standard of care alone in improving retention in care and promoting adherence to treatment among HIV clients on treatment at 2, 4, and 6 months. Retention in care shall be defined as the proportion of clients who had started on ART, enrolled in the study, and attended clinic visits at the second, fourth, or sixth months. There is no gold standard in the measurement of adherence because the majority of the tools currently used cannot meet all the features of an ideal tool [25]. However, a multi-method tool is recommended and can include self-report and different combinations of other tools including pill count, the pill identification test (PIT) and visual analog scale (VAS), electronic methods, and drug levels. Therefore, a composite adherence measure shall be used in this study to reduce the errors associated with using a single adherence measure [25]. The adherence score shall be built on four adherence measures including a self-report questionnaire with
4 items, supplement of pill pick up from pharmacy refill records, a PIT consisting of 5 items, and a 30-day VAS. Adherence shall first be estimated by each measure and classified into three categories as high, moderate, and low before finally combining the results of the four measures into the composite adherence score.

\section{Secondary Objectives}

The secondary objectives include comparing health outcomes such as weight, BMI, opportunistic infections such as tuberculosis, and quality of life. These comparisons will be performed between the groups at baseline and at 2, 4, and 6 months. In addition, the viral load will be determined for participants who have been receiving ART for at least 6 months to ascertain virologic suppression.

\section{Outcome Measures}

\section{Primary outcomes}

The primary outcomes will be retention in care and adherence to treatments, measured using self-reports (supplemented by PIT, pharmacy refill data, and VAS; Table 1).

Table 1. Overview of outcome measures.

\begin{tabular}{|c|c|c|c|c|}
\hline Outcome measures & Scale & Type & Measure & Analysis method \\
\hline \multicolumn{5}{|l|}{ Primary } \\
\hline Retention at 2, 4, and 6 months & Nominal & Binary & Number retained in care & Risk ratio \\
\hline \multicolumn{5}{|c|}{ Adherence at baseline and at 2,4 , and 6 months } \\
\hline Self-report & Ordinal & Binary & $\%$ adherence in last month $>95 \%$ & Risk ratio \\
\hline Pill identification test & Ordinal & Binary & $\%$ of pills identified $>95 \%$ & Risk ratio \\
\hline $\mathrm{PRD}^{\mathrm{a}}$ & Ordinal & Binary & $\%$ of complete refills $>95 \%$ & Risk ratio \\
\hline $\mathrm{VAS}^{\mathrm{b}}$ & Ordinal & Binary & VAS percentage $>95 \%$ & Risk ratio \\
\hline \multicolumn{5}{|l|}{ Secondary } \\
\hline Weight & Ratio & Continuous & Change in weight & $t$ test \\
\hline BMI & Ratio & Continuous & Change in BMI & $t$ test \\
\hline Viral load & Ratio & Continuous & Change in viral load & $t$ test \\
\hline $\mathrm{OIs}^{\mathrm{c}}\left(\mathrm{AFB}^{\mathrm{d}}\right.$ diagnosis $)$ & Nominal & Binary & $\begin{array}{l}\text { Occurrence of new OI (AFB } \\
\text { positive smear) }\end{array}$ & Chi-square test \\
\hline Mortality & Nominal & Binary & All deaths & Chi-square test \\
\hline Satisfaction with care & Ordinal & Categorical & Change in satisfaction scores & $t$ test \\
\hline $\mathrm{QoL}^{\mathrm{e}}$ & Ordinal & Categorical & Change in QoL scores & $t$ test \\
\hline \multicolumn{5}{|l|}{ Overall } \\
\hline & Nominal & Binary & Number retained in each group & Risk ratio \\
\hline & Ordinal & Binary & $\begin{array}{l}\text { Change in composite adherence } \\
\text { measure }\end{array}$ & Risk ratio \\
\hline
\end{tabular}

\footnotetext{
${ }^{a} P R D$ : pharmacy refill data.

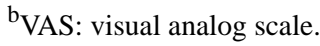

${ }^{\mathrm{c}} \mathrm{OI}$ : opportunistic infection.

${ }^{\mathrm{d}} \mathrm{AFB}$ : acid-fast bacilli.

${ }^{\mathrm{e}} \mathrm{QoL}$ : quality of life.
} 


\section{Secondary Outcomes}

The secondary endpoints shall comprise clinical (weight and BMI), biological (viral load and AFB diagnosis), quality of life (measured with the self-functioning-12 quality of life assessment form), and all-cause mortality. Treatment discontinuation, reasons for discontinuation, and risk factors of treatment discontinuation shall also be determined. The trial endpoints are summarized in Table 1.

\section{Duration}

The trial shall run for 6 months. Outcome assessments will be conducted at baseline, 2 months, 4 months, and 6 months.

\section{Sample Size Determination}

The sample size determination is based on the test of the null hypothesis that the rates of adherence to ART in the intervention and control groups are equal. The primary measure of effect is the rate of adherence to ART as measured by using the VAS over 6 months. Given the information from previous studies $[10,11]$, it is estimated that approximately $60 \%$ of participants would maintain adherence $\geq 95 \%$ without intervention and that the interventions would help $82 \%$ of the participants maintain $\geq 95 \%$ adherence. A two-sided test is assumed where an effect in either direction will be interpreted at a significance level of $5 \%$. The study will have a power of $80 \%$ to yield a statistically significant result using a chi-square test (assuming an intention-to-treat principle for the analysis) of the relative risk at an alpha level of $5 \%$ to detect a $25 \%$ difference $(60 \%$ vs $85 \%)$.

The formula for calculating the sample size for randomized controlled trials by Chan [26] is used.

$$
\mathrm{n}(\text { size per group })=\mathrm{cX}\left[\frac{\mathrm{p} 1(1-\mathrm{p} 1)+\mathrm{p} 2(1-\mathrm{p} 2)}{(\mathrm{p} 1-\mathrm{p} 2)^{2}}\right]
$$

where $\mathrm{c}=7.9$ for $80 \%$ power and $\mathrm{p} 1$ and $\mathrm{p} 2$ are the proportion estimates $(60 \%$ for the control group and $85 \%$ for the intervention group).

$$
\begin{gathered}
\mathrm{n}\left(\text { size per group) }=7.9 \times\left[\frac{0.6(1-0.6)+0.85(1-0.85)}{(0.6-0.85)^{2}}\right]\right. \\
\mathrm{n} \text { (size per group) }=46.5
\end{gathered}
$$

It is assumed that $10 \%$ of the participants shall drop out of the study due to loss to follow-up and mortality. A total of 52 participants shall be randomized to each arm to allow for the $10 \%$ drop out. Therefore, the required sample size will be 156 $(52 \times 3=156)$ participants.

\section{Analysis Plan}

The CONSORT (Consolidated Standards of Reporting Trials) guidelines will be used in reporting the results. The biostatistician shall be blinded to the group allocation. The process of client selection and flow throughout the study will be summarized using a flow diagram. The analysis of client demographics and outcome variables shall be summarized using descriptive summary measures, expressed as mean (standard deviation) or median (range) for continuous variables and number (percentages) for categorical variables. All outcomes shall be analyzed using an intention-to-treat principle where data from participants shall be analyzed according to the group to which they were randomized even if they do not receive the allocated intervention. Missing data shall be handled using the multiple-imputation method. The student $t$ test and analysis of variance for comparing group means will be used. All statistical tests shall be performed using two-sided tests at the 5\% level of significance. The Bonferroni method shall be used to adjust the level of significance for testing of secondary outcomes to keep the overall level at an alpha of 5\%. For all group comparisons, the results shall be expressed as an effect (or risk ratio for binary outcomes), corresponding two-sided $95 \%$ confidence intervals, and associated $P$ values. $P$ values shall be reported to three decimal places with values less than .001 reported as <.001. Adjusted analyses using baseline variables shall be performed using regression techniques to determine the continuing influence of key baseline characteristics on the outcomes. The Kaplan-Meier survival analysis will be used for timed variables like mortality. All analyses will be performed using SPSS version 25.0 (IBM Corp) for Windows. Adherence will be measured both as a continuous outcome (change in adherence) and as a binary outcome (ie, adherent, $95 \%$ of pills taken, or nonadherent, $<95 \%$ of pills taken) following the composite adherence score method. Adherence data shall be handled in a number of ways, reported as the number of doses respected, and shall be combined into a composite score. The effects of the intervention shall be reported on all the measures of adherence used and will be compared for discrepancies.

\section{Patient and Public Involvement}

This study was conceived and designed to address gaps in the care and support available to people living with HIV/AIDS who are receiving ART. The intervention was designed with the active involvement of patients with HIV, their caregivers, and health workers through focus group discussions. Our randomized controlled trial offers participants the opportunity to provide feedback regarding the burden of the intervention through focus group discussions involving a cross-section of participants.

\section{Ethics and Dissemination}

Ethical clearance for this trial was obtained from the Institutional Review Board of the Faculty of Health Sciences of the University of Buea in Cameroon (Reference number: 2018/147/UB/SG/IRB/FHS). Administrative authorization was obtained from the Regional Delegation of the Ministry of Public Health for the South West Region and the District Health Services. The purpose of the study and the role of the participants will be well explained in the consent form to the participants and participation shall only take place after the participant has read and signed the informed consent forms voluntarily. The informed consent shall include signed permission to consult the client's medical records over the duration of the study.

Dissemination efforts shall target individuals and institutions that will have the most impact on local, national, and international HIV and AIDS policies. Therefore, dissemination plans shall include the presentation of research findings in seminars, conferences, and scientific publications in 
peer-reviewed journals. A summary of the findings will be made available to participants.

\section{Results}

Recruitment of participants in the trial took place in May and June 2018. Recruited participates were randomly allocated to the intervention and control arms at the start of the intervention in July 2018.

A total of 160 participants have been recruited and randomly allocated into the intervention and control groups. Participants were allocated into 3 groups (52 in the once weekly SMS, 55 in the twice weekly SMS, and 53 in the control). Data collection and analysis are ongoing, and statistical results will be available by the end of August 2019.

\section{Discussion}

Since the launch of the fast track approach to end the AIDS epidemic by 2030, Cameroon is far from reaching the $90-90-90$ treatment target in 2020 , whereby $90 \%$ of people living with HIV know their HIV status, $90 \%$ of people who know their HIV-positive status are accessing treatment, and $90 \%$ of people on treatment have suppressed viral loads [27]. Only 58\% of people living with HIV know their HIV status, $37 \%$ of those living with HIV are on treatment, and $19 \%$ of these patients are virally suppressed 2 years from the 2020 treatment target in Cameroon [27].

New strategies to end the AIDS epidemic as a public health threat by 2030 are being instituted throughout most national HIV control programs [28]. Recent advances in the fast-track approach to HIV and AIDS in Cameroon has been the recommendation of offering to every client presenting to a clinic for any medical consultation to pass the HIV screening test and to allow as many people to know their HIV status [29]. In addition, the government of Cameroon through the Ministry of Public Health has instituted new directions by placing the systematic treatment of HIV under the strategy called test and treat (screening and treatment) [29]. This new strategy requires that any person screened and confirmed positive for HIV is directly placed on antiretroviral treatment. Proper retention in care and a high level of adherence are needed to sustain lower viral load counts and reduce chances of drug resistance that might result from treatment defaulting. More people will be accessing ART services as a result of enhanced HIV testing. There is a high need to devise strategies that would lead to improved retention in care and sustained adherence to treatment.

In this light, the benefits of mobile phone SMS in improving and promoting health outcomes are warranted, particularly in this era of increased uptake and use of mobile phone devices. However, there is a paucity of economic data to support the use of mHealth behavioral interventions in low- and low-middle-income countries [30].

Improving retention in care and promoting adherence to treatments can play a key role in reducing the morbidity and mortality associated with HIV and tuberculosis diseases. The occurrence of drug-resistant strains and the waste of medication in health systems can be adequately managed. Findings generated from this trial may be generalizable to other chronic illnesses requiring lifelong treatments.

A major ethical concern is the harm that might be caused to participants due to the accidental disclosure of their disease status. This possibility shall be properly explained to the participant, even though our text messages shall neither disclose status nor make mention of medication but shall rather act as a reminder of health. Loss of privacy and confidentiality are not foreseeable problems in the study. The intervention shall be an addition to an already existing system in Cameroon where mobile operators deliver messages to their clients for business purposes. The text messages shall be delivered to the participants' mobile phones by the research team using phones with prepaid airtime.

The content of the SMS was developed following the construct of the health belief model of behavior change. Furthermore, the intervention shall focus on the health belief model. Collected data shall be used to test the efficacy of the SMS reminder as a cue to action. This study is timely, as it is based on this strategy to improve and promote ART and tuberculosis treatment adherence and retention in care in Cameroon. Furthermore, the study will enhance our understanding of the extent that mHealth intervention promotes healthy behaviors and support psychosocial well-being among patients receiving treatment for chronic diseases. The study shall also investigate the effect of tuberculosis coinfection on the health outcomes of HIV clients. Therefore, the study will contribute to an improved understanding of which type of text message frequency can be feasible when, why, and for whom.

\section{Acknowledgments}

We are grateful to the directors and staff of Buea Regional Hospital, Limbe Regional Hospital, Tiko Central Clinic, and Muyuka District Hospital for assistance in the trial design. We are also thankful to all the patients and their caregivers who participated in the development of the intervention's content. This is part of a PhD thesis conducted on "Retention in Care and Adherence to Treatment in HIV/Tuberculosis Co-infection in Fako Division, Cameroon" at the Faculty of Health Sciences of the University of Buea in Cameroon.

\section{Authors' Contributions}

EAT and DSN conceived the study. EAT, DSN, NJCA, and TNN helped draft the manuscript. EAT, DSN, NJCA, and TNN designed the trial. All authors read and approved the final manuscript. 


\section{Conflicts of Interest}

None declared.

\section{References}

1. Thompson MA, Aberg JA, Cahn P, Montaner JSG, Rizzardini G, Telenti A, International AIDS Society-USA. Antiretroviral treatment of adult HIV infection: 2010 recommendations of the International AIDS Society-USA panel. JAMA 2010 Jul 21;304(3):321-333. [doi: 10.1001/jama.2010.1004] [Medline: 20639566]

2. The Antiretroviral Therapy in Lower Income Countries (ART-LINC) Collaboration, ART Cohort Collaboration (ART-CC) groups. Mortality of HIV-1-infected patients in the first year of antiretroviral therapy: comparison between low-income and high-income countries. Lancet 2006 Mar;367(9513):817-824. [doi: 10.1016/S0140-6736(06)68337-2]

3. Prevention gap report. UNAIDS. 2016. URL: http://www.unaids.org/sites/default/files/media asset/ 2016-prevention-gap-report en.pdf [accessed 2017-02-20]

4. List of countries by number of mobile phones in use. Wikipedia. 2016. URL: https://en.wikipedia.org/w/index. php?title=List of countries by number of mobile phones in use\&oldid=808413235 [accessed 2016-11-09]

5. The world factbook. Central Intelligence Agency. 2016. URL: https://www.cia.gov/library/publications/the-world-factbook/ geos/cm.html [accessed 2016-11-09]

6. Lester RT, Mills EJ, Kariri A, Ritvo P, Chung M, Jack W, et al. The HAART cell phone adherence trial (WelTel Kenya1): a randomized controlled trial protocol. Trials 2009 Sep 22;10:87 [FREE Full text] [doi: 10.1186/1745-6215-10-87] [Medline: $\underline{19772596]}$

7. De Costa A, Shet A, Kumarasamy N, Ashorn P, Eriksson B, Bogg L, HIVIND study team. Design of a randomized trial to evaluate the influence of mobile phone reminders on adherence to first line antiretroviral treatment in South India--the HIVIND study protocol. BMC Med Res Methodol 2010 Mar 26;10:25 [FREE Full text] [doi: 10.1186/1471-2288-10-25] [Medline: 20346136]

8. Mbuagbaw L, Thabane L, Ongolo-Zogo P, Lester RT, Mills E, Volmink J, et al. The Cameroon mobile phone SMS (CAMPS) trial: a protocol for a randomized controlled trial of mobile phone text messaging versus usual care for improving adherence to highly active anti-retroviral therapy. Trials 2011 Jan 07;12:5 [FREE Full text] [doi: 10.1186/1745-6215-12-5] [Medline: 21211064]

9. Resource needs for an expanded response to AIDS in low- and middle-income countries. UNAIDS. 2005. URL: http://www. unaids.org/sites/default/files/media asset/jc1255 resource needs en 0.pdf [accessed 2017-02-22]

10. Lester RT, Ritvo P, Mills EJ, Kariri A, Karanja S, Chung MH, et al. Effects of a mobile phone short message service on antiretroviral treatment adherence in Kenya (WelTel Kenya1): a randomised trial. Lancet 2010 Nov;376(9755):1838-1845. [doi: 10.1016/s0140-6736(10)61997-6]

11. Mbuagbaw L, Thabane L, Ongolo-Zogo P, Lester RT, Mills EJ, Smieja M, et al. The Cameroon Mobile Phone SMS (CAMPS) trial: a randomized trial of text messaging versus usual care for adherence to antiretroviral therapy. PLoS One 2012;7(12):e46909 [FREE Full text] [doi: 10.1371/journal.pone.0046909] [Medline: 23236345]

12. Nsagha DS, Lange I, Fon PN, Nguedia Assob JC, Tanue EA. A randomized controlled tial on the usefulness of mobile text phone messages to improve the quality of care of HIV and AIDS patients in Cameroon. Open AIDS J 2016;10:93-103 [FREE Full text] [doi: 10.2174/1874613601610010093] [Medline: 27583062]

13. Mukund Bahadur KC, Murray PJ. Cell phone short messaging service (SMS) for HIV/AIDS in South Africa: a literature review. Stud Health Technol Inform 2010;160(Pt 1):530-534. [Medline: 20841743]

14. Mbuagbaw L, van der Kop ML, Lester RT, Thirumurthy H, Pop-Eleches C, Ye C, et al. Mobile phone text messages for improving adherence to antiretroviral therapy (ART): an individual patient data meta-analysis of randomised trials. BMJ Open 2013 Dec 17;3(12):e003950 [FREE Full text] [doi: 10.1136/bmjopen-2013-003950] [Medline: 24345901]

15. Leong KC, Chen WS, Leong KW, Mastura I, Mimi O, Sheikh MA, et al. The use of text messaging to improve attendance in primary care: a randomized controlled trial. Fam Pract 2006 Dec;23(6):699-705. [doi: 10.1093/fampra/cml044] [Medline: $\underline{16916871]}$

16. Badawy SM, Cronin RM, Hankins J, Crosby L, DeBaun M, Thompson AA, et al. Patient-centered eHealth interventions for children, adolescents, and adults with sickle cell disease: systematic review. J Med Internet Res 2018 Jul 19;20(7):e10940 [FREE Full text] [doi: 10.2196/10940] [Medline: $\underline{\text { 30026178] }}$

17. Majeed-Ariss R, Baildam E, Campbell M, Chieng A, Fallon D, Hall A, et al. Apps and adolescents: a systematic review of adolescents' use of mobile phone and tablet apps that support personal management of their chronic or long-term physical conditions. J Med Internet Res 2015 Dec 23;17(12):e287 [FREE Full text] [doi: 10.2196/jmir.5043] [Medline: 26701961]

18. Badawy SM, Kuhns LM. Texting and mobile phone app interventions for improving adherence to preventive behavior in adolescents: a systematic review. JMIR Mhealth Uhealth 2017 Apr 19;5(4):e50 [FREE Full text] [doi: 10.2196/mhealth.6837] [Medline: 28428157]

19. Badawy SM, Barrera L, Sinno MG, Kaviany S, O'Dwyer LC, Kuhns LM. Text messaging and mobile phone apps as interventions to improve adherence in adolescents with chronic health conditions: a systematic review. JMIR Mhealth Uhealth 2017 May 15;5(5):e66 [FREE Full text] [doi: 10.2196/mhealth.7798] [Medline: 28506955] 
20. The HIV/TB co-infection mobile phone SMS trial. Pan African Clinical Trials Registry. 2018. URL: https://pactr.samrc.ac.za/ TrialDisplay.aspx?TrialID=3035 [accessed 2018-02-21]

21. Cameroon population. Country Meters. URL: http://countrymeters.info/en/Cameroon [accessed 2016-11-17]

22. Ministry of Public Health. Cameroon population-based HIV impact assessment results. CAMPHIA 2018:1-10.

23. National guideline on the prevention and management of HIV in Cameroon. Ministry of Public Health. 2015. URL: https:/ /www.childrenandaids.org/sites/default/files/2018-05/Cameroon Nat\%20Guidelines\%20HIV 2015.pdf [accessed 2017-09-18]

24. Rosenstock IM, Strecher VJ, Becker MH. The health belief model HIV risk behavior change. In: DiClemente RJ, Peterson JL, editors. Preventing AIDS Theories and Methods of Behavioral Interventions. New York: Plenum Press; 1994:5-24.

25. Steel G, Nwokike JI, Mp J. Development of a multi-method tool to measure ART adherence in resource constrained settings: the South Africa experience. Rational Pharmaceutical Management Plus Program. 2007. URL: https://pdfs. semanticscholar.org/7d54/8ccab52965efcd3f9e14d80aab7aa05d7bfd.pdf [accessed 2017-10-20]

26. Chan YH. Randomised controlled trials (RCTs)--sample size: the magic number? Singapore Med J 2003 Apr;44(4):172-174. [Medline: 12952027$]$

27. Ending AIDS: progress towards the 90-90-90 targets. UNAIDS. 2017. URL: https://www.unaids.org/sites/default/files/ media asset/Global AIDS update 2017 en.pdf [accessed 2017-09-20]

28. On the fast-track to end AIDS. UNAIDS. 2015. URL: http://www.unaids.org/en/resources/documents/2015/ UNAIDS PCB37 15-18 [accessed 2017-10-02]

29. Who's afraid of the new routine HIV screening? CamerounWeb. URL: http://www.cameroonweb.com/CameroonHomePage/ NewsArchive/Who-s-afraid-of-the-new-routine-HIV-screening-378601 [accessed 2016-11-09]

30. Iribarren SJ, Cato K, Falzon L, Stone PW. What is the economic evidence for mHealth? a systematic review of economic evaluations of mHealth solutions. PLoS One 2017;12(2):e0170581 [FREE Full text] [doi: 10.1371/journal.pone.0170581] [Medline: 28152012]

\author{
Abbreviations \\ ART: antiretroviral therapy \\ CONSORT: Consolidated Standards of Reporting Trials \\ mHealth: mobile health \\ PIT: pill identification test \\ PRD: pharmacy refill data \\ VAS: visual analog scale
}

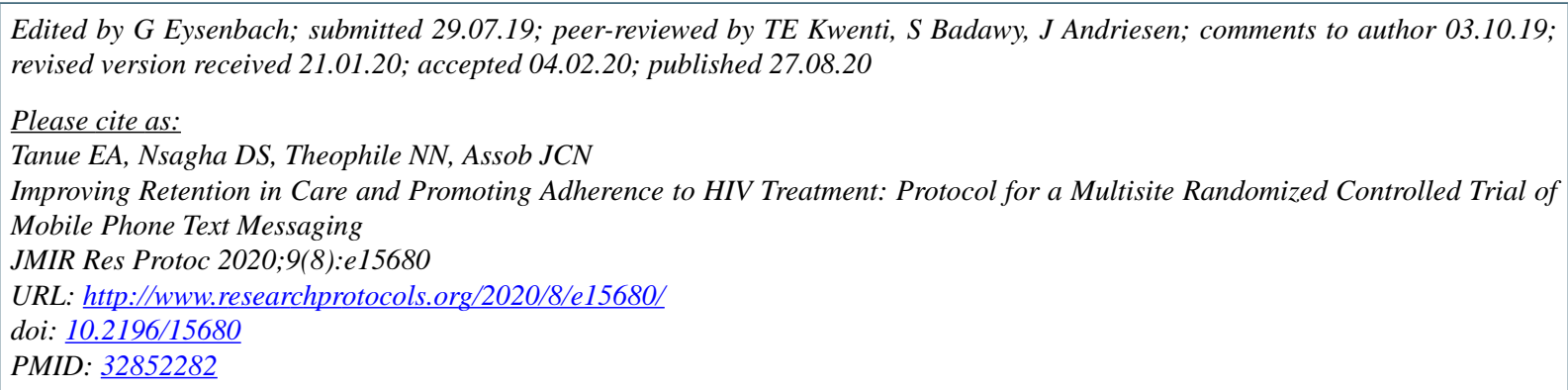

CElvis Asangbeng Tanue, Dickson Shey Nsagha, Nana Njamen Theophile, Jules Clement Nguedia Assob. Originally published in JMIR Research Protocols (http://www.researchprotocols.org), 27.08.2020. This is an open-access article distributed under the terms of the Creative Commons Attribution License (https://creativecommons.org/licenses/by/4.0/), which permits unrestricted use, distribution, and reproduction in any medium, provided the original work, first published in JMIR Research Protocols, is properly cited. The complete bibliographic information, a link to the original publication on http://www.researchprotocols.org, as well as this copyright and license information must be included. 